\title{
The Feasibility of Opening Communities -- Based on the Wiedemann Car-Following Model
}

\author{
Xiao-Yan Cao ${ }^{1}$, Bing-Qian Liu ${ }^{1}$, Bao-Ru Pan $^{1} \&$ Yuan-Biao Zhang ${ }^{1,2,3}$ \\ ${ }^{1}$ Mathematical Modeling Innovative Practice Base, Jinan University, Zhuhai Campus, Zhuhai, China \\ ${ }^{2}$ Packaging Engineering Institute, Jinan University, Zhuhai Campus, Zhuhai, China \\ ${ }^{3}$ Key Laboratory of Product Packaging and Logistics of Guangdong Higher Education Institutes, Jinan University, \\ Zhuhai Campus, Zhuhai, China \\ Correspondence: Yuan-Biao Zhang, Packaging Engineering Institute, Jinan University, Zhuhai Campus, Zhuhai, \\ China. E-mail: zybt@jnu.edu.cn
}

Received: June 2, 2018

doi:10.5539/cis.v11n3p57
Accepted: June 28, $2018 \quad$ Online Published: July 2, 2018

URL: https://doi.org/10.5539/cis.v11n3p57

\begin{abstract}
With the accelerating development of urbanization in China, the increasing traffic demand and large scale gated communities have aggravated urban traffic congestion. This paper studies the impact of communities opening on road network structure and the surrounding road capacity. Firstly, we select four indicators, namely average speed, vehicle flow, average delay time, and queue length, to measure traffic capacity. Secondly, we establish the Wiedemann car-following model, then use VISSIM software to simulate the traffic conditions of surrounding roads of communities. Finally, we take Shenzhen as an example to simulate and compare the four kinds of gated communities, axis, centripetal and intensive layout, and we also analyze the feasibility of opening communities.
\end{abstract}

Keywords: gated community, road network structure, Wiedemann car-following model, VISSIM

\section{Introduction}

With the expansion of Chinese cities scale, gated communities have become a major factor hindering the development of urban road traffic. The question of whether or not gated communities need to be opened has attracted widespread attention from social public. It is a focal point for solving the problem of urban traffic congestion.

Baycan $T$ believes that gated communities pose a threat to the sustainability of natural resources (Akgün, A. A.\& Baycan, T, 2012).Xu and Yang analyzed the impact of gated communities on the development of urban space, and then proposed the deficiencies of urban planning and design in China, and gave some solutions (Xu \& Yang, 2010).Li and He studied the differences between gated communities and open cells, then pointed out that the opening of gated communities needs to grasp the appropriate degree(Li Jun \& He Lian, 2007).Li described the characteristics of gated communities, and then through simulation found that the opening of gated communities can improve accessibility of road networks and reduce travel costs(Li Xiangpeng, 2014).Bao and Zhang analyzed the development of gated communities, and believed that this urban construction model caused urban traffic congestion (Bao Yipeng \& Zhang Xinyi, 2016);Wei discussed on the characteristics of the evolution of residential communities, enumerated a series of reasons for blocking communities, and combined the successful cases of the transformation of communities model in Western countries, and put forward specific ideas for opening gated communities (Wei, 2016); Qiang held that gated communities has caused damage to the public space, which affected the construction of public traffic road networks and were detrimental to the development of urban traffic (Qiang Naishe, 2016);Tang studied the feasibility and practical obstacles to the road publication of gated communities from a legal perspective (Tang Kai, 2017);He and Lan established the traffic diversion network model and analyzed the impact of gated communities on urban traffic before and after they were opened, and concluded that whether the community to open or not depends on factors such as the size of the area, geographic location, and surrounding road structure. (He Liang \& Lan Yang, 2017).

This paper selected four indicators of average speed, vehicle flow, average delay time, and queue length to measure traffic capacity. Then we established the Wiedemann following model to analyze the traffic conditions of surrounding roads before and after the opening of gated communities. In the paper, we took Shenzhen as an 
example to study the effects of four different structures of communities after opening, such as patch layout, axis layout, centripetal layout, and intensive layout.

\section{Method}

There are many indicators for assessing the capacity of roads, such as the average speed of vehicles, vehicle flow passing a point on the road, travel time, delay in the itinerary, V/C and etc. ( $\mathrm{Li}$, Zhu et al, 2017). It is obviously impractical and insignificant to analyze all evaluation indicators. Therefore, this paper selects the most representative four indicators to illustrate the impact of community opening on the surrounding road traffic.

\subsection{Determine the Evaluation Indexes}

\subsubsection{Average Speed}

The average speed $\bar{v}$ refers to the average speed of all vehicles passing through a section of road within a certain period of time. The average value is not only related to each vehicle, but also changes with the change of statistical time. Its mathematical expression is similar to the following:

$$
\bar{v}=\frac{\int_{t=0}^{T} \frac{1}{N(t)} \sum_{i=1}^{N(t)} v_{i} d t}{T}
$$

Where, $\bar{v}$ indicates the average value of the speed of all vehicles passing through a certain road within a certain period of time; $T$ indicates the total statistical time; $N(t)$ indicates the number of vehicles on the road at time $t$; $v_{i}$ indicates the speed of the $i$-th car at time $t$.

\subsubsection{Vehicle Flow}

Vehicle flow $Q$ refers to the number of vehicles passing through the designated section of the road per unit time. If an intersection is specified as a measurement point, the maximum traffic volume and the average traffic volume through this location within a specified time can be counted.

If it is impossible to count the average traffic volume of a given section of a road, it can also be replaced by the following method within the allowable range of error:

$$
q=\frac{N(t)}{L}
$$

Where, $N(t)$ is the number of vehicles on the road at time $t, L$ is the road length. $q$ is the vehicle density on the road at time $t$.

The average speed of all vehicles and vehicle flow $Q$ on the road at that time are calculated by

$$
\begin{gathered}
\bar{v}(t)=\frac{1}{N(t)} \sum_{i=1}^{N(t)} v_{i} \\
Q=q \overline{\mathcal{V}}(t)
\end{gathered}
$$

\subsubsection{Average Delay Time}

In urban road traffic networks, due to the existence of intersections, vehicles often suffer from different extent delays due to factors such as deceleration of the front vehicle and waiting for signals. Obviously, the length of the delay time can visually reflect the traffic capacity of the intersection. Intersections of roads can directly affect the fluency of local traffic. Therefore, the intersection delay time is an important indicator in urban traffic management, traffic planning, and traffic economic analysis (Gao Yangbin, 2004).

With individual vehicles as the unit, the average delay time $\bar{d}$ is calculated by tracking and recording the driving condition of the vehicle using the average delay calculation method for real-time measurement (Wang, Wu et al,2017), its calculated formula is as follows:

$$
\begin{aligned}
& d_{i}=\left|t_{i}-t_{0}\right| \\
& \bar{d}=\sum_{i=1}^{N} d_{i} / N
\end{aligned}
$$


Where, $d_{i}$ indicates the delay time for the $i$-th car to cross a intersection; $t_{i}$ indicates the actual consumption time for the $i$-th car to cross the intersection; $t_{0}$ indicates the time required for the vehicle to pass through the intersection in a free-flow state; $\bar{d}$ indicates the average delay time at the intersection; $N$ indicates the total number of vehicles passing the intersection during the analysis period.

\subsubsection{Queue Length}

Queue length $L_{n}$ is another important indicator for studying traffic congestion. Traffic jams, signal lights and other conditions will cause the vehicle to stop and affect traffic conditions. Therefore, the queue length can also directly reflect road traffic conditions. The calculated formula is as follows:

$$
\begin{gathered}
L_{n}=D_{n-1}+2 Q_{n} \\
D_{n}=\left\{\begin{array}{cc}
D_{n-1}+Q_{n} T+C_{a} R-G S & D_{n-1}+Q_{n} T+C_{a} R-G S>0 \\
0 & D_{n-1}+Q_{n} T+C_{a} R-G S \leq 0
\end{array}\right.
\end{gathered}
$$

Where, $L_{n}$ indicates the number of vehicles in the queue, that is, the queue length; $Q_{n}$ indicates the duration of the traffic light cycle; $T$ indicates the road capacity, that is, the limit value of the vehicle operation that can be carried by roads per unit time; $R$ indicates the red light duration, $G$ indicates the green light duration; $S$ indicates saturated vehicle flow; $D_{n}$ is an intermediate value that is continuously iterated from the first $n-1$ cycles.

\subsection{Wiedemann Car-Following Model}

The Wiedemann model is based on the vehicle distance and vehicle speed to determine the driving psychology and behavior of drivers. The driver adjusts the following speed by the relative movement between the front and rear cars, including speed and distance (excitation), and these incentives can only be perceived and reacted by the driver if they exceed a certain threshold ( $\mathrm{Li}$, Song et al,2013).

The specific threshold value refers to the critical value that causes the driver's driving behavior to change, that is, the difference between the front and rear vehicle speed and the vehicle distance. The driving behavior is divided into several categories based on different vehicle speed differences and distances. The details are as follows:

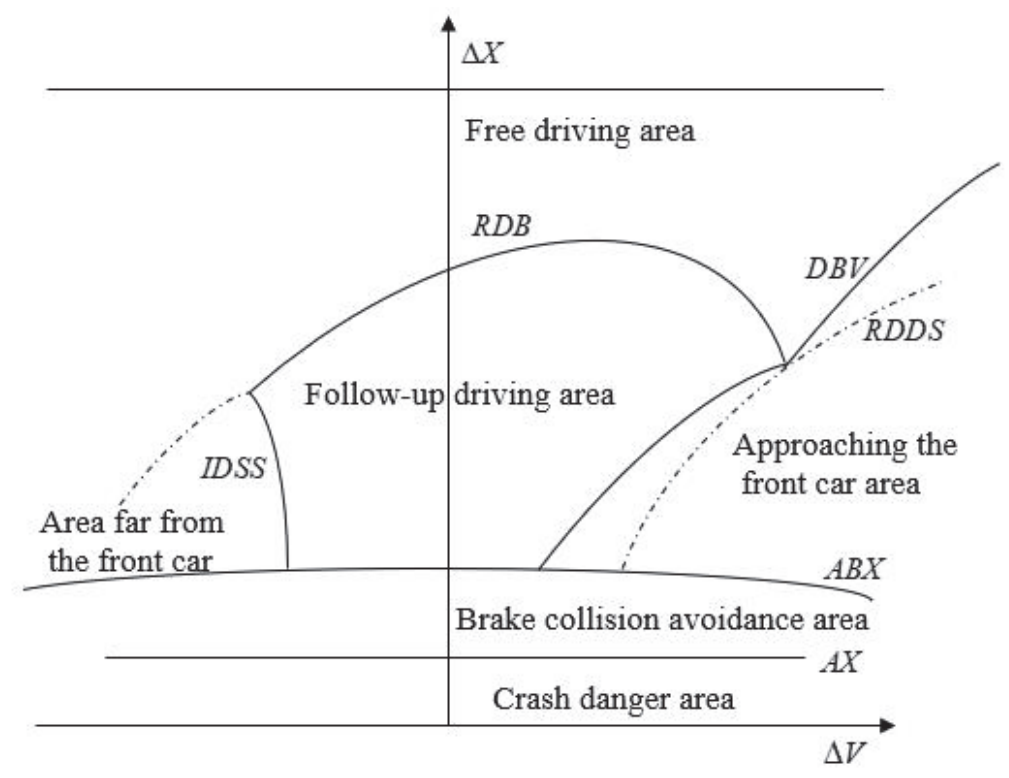

Figure 1. The behavior domain of Wiedemann model

In the above figure 1, the abscissa $\Delta V$ indicates the front and rear car speed difference; the ordinate $\Delta X$ indicates the front and rear car distance; $A X$ indicates the desired distance when the vehicle is stationary, which is slightly greater than the length of the front vehicle. If the current follow-up distance of the rear vehicle is lower than $A X$, then the following vehicle will enter the danger area of the collision and collision may occur. $A B X$ indicates that the minimum following distance between the front and rear cars should be maintained when the speed difference between the front and rear cars is small. If the distance between the front and rear cars is smaller than this value, 
the follower vehicle will automatically enter the braking state and thus avoid colliding with the front car. This value is not only related to $\mathrm{A}$, but also directly related to the instantaneous speed of the front and rear cars. $R D B$ is the threshold that the driver of the rear vehicle can realize that the vehicle distance becomes larger during the car-following process. Above this threshold, the driver can realize that he is getting farther and farther from the vehicle. $D B V$ is the threshold that the driver of the rear vehicle can realize that the vehicle speed becomes larger than the front vehicle when the distance between the front and rear is large. Above this threshold, the driver can realize that the distance between two vehicles is decreasing. $R D D S$ is the threshold that the driver of the rear vehicle can realize that the vehicle speed becomes slightly larger than the front vehicle when the distance between the front and rear is small. Above this threshold, the driver realizes that he is slowly approaching a very close car and the distance between cars is slowly decreasing. IDSS is the threshold that the driver of the rear vehicle can realize that the vehicle speed becomes slightly smaller than the front vehicle when the distance between the front and rear is small. Above this threshold, the driver realizes that he is slowly away from a very close car and the distance between cars is slowly increasing.

This paper uses VISSIM traffic simulation software to implement this model. The software can analyze the traffic conditions under various conditions and can effectively evaluate the urban road traffic problems (Liu \& Wang, 2006).

\subsubsection{The Free Driving Area}

When the follower vehicle enters the free driving area, that is, the speed difference and the vehicle distance satisfy the conditions greater than $R D B$ and $D B V$, the vehicle will accelerate at the maximum acceleration $b_{\max }$ to reduce the distance between the front and rear cars.

$$
b_{\max }=\lambda_{b}-\beta_{b} \cdot v_{n}
$$

Where, $\lambda_{b}$ is the parameter to be calibrated, which limits the maximum range of maximum acceleration. This paper takes the most common value of $3.5 ; \beta_{b}$ is the parameter to be calibrated, which represents the sensitivity of the maximum acceleration followed by the speed change of the vehicle. This paper takes the most common value of $0.0875 ; v_{n}$ indicates the instantaneous speed of the rear vehicle.

When the follower vehicle reaches the desired speed, the driver will perform a habitual unconscious acceleration/deceleration to indicate that the driver of the follower vehicle has inaccurately controlled acceleration and deceleration, which can simulate the acceleration/deceleration process with a random probability. \pm accel indicates the acceleration of the driver's habitual acceleration and deceleration:

$$
\text { accel }=\lambda_{\text {accel }} \cdot \text { rand }
$$

Where, $\lambda_{\text {accel }}$ is the acceleration and deceleration multiplier coefficient, this paper takes the most common value of 0.1 ; rand is a non-negative random value that simulates the driver's inaccurately controlled acceleration and deceleration behavior.

\subsubsection{The Follow-up Driving Area}

When the speed difference and the car distance of the follower vehicle enter the follow-up driving area formed by $D B V 、 R D B 、 I D S S 、 A B X$, the rear car driver follows the front car and constantly adjusts the current vehicle speed in accordance with the change of the front car speed and the vehicle distance. From the different behavioral domains into the follow-up driving area, different adjustment methods will be adopted.

When the follower vehicle follows the $A B X$ curve into the follow-up driving area, because the distance between the rear car and the front car is still small, the rear car driver can only maintain the original speed or the customary deceleration to maintain the safety distance. So the acceleration at this time is -accel .

When the follower vehicle follows the $D B V$ curve into the follow-up driving area, the distance between the rear car and the front car is larger and the rear car is faster. According to the psychological experience, the driver of the rear car will perform deceleration adjustment at this time. So the acceleration at this time is -accel.

When the follower vehicle follows the $R D B 、 I D S S$ curve into the follow-up driving area, the rear car speed is smaller than the front car, and the rear car driver will habitually increase the speed. So the acceleration at this time is accel. 


\subsubsection{Approaching the Front Car Area}

When the speed difference and the car distance of the follower vehicle enter the approaching the front car area formed by $A B X, D B V 、 R D D S$, the vehicles are in the approaching state, and the front vehicle decelerates and the rear vehicle accelerates. In order to avoid colliding with the preceding vehicle, the rear vehicle driver will perform corresponding deceleration measures:

$$
b_{n}=\frac{1}{2} \cdot \frac{(\Delta v)^{2}}{A B X-\left(\Delta v-L C_{n-1}\right)}+b_{n-1}
$$

Where, $b_{n} 、 b_{n-1}$ indicate the acceleration of the rear and front cars respectively. $\Delta v$ indicates the speed difference

between the front and rear cars. $A B X$ is the minimum follow-up distance threshold introduced above. $L C_{n-1}$ indicates the length of the front vehicle.

\subsubsection{Area Far from the Front Car}

When the speed difference and the car distance of the follower vehicle enter the area far from the front car formed by $A B X, I D D S$, the rear vehicle speed is much smaller than the front vehicle and the vehicle will be in a leaving state. The rear driver shifts from a car-following state to a free-driving state where the vehicle accelerates with maximum acceleration:

$$
b_{\max }=\lambda_{b}-\beta_{b} \cdot v_{n}
$$

Where, $\lambda_{b}$ is the parameter to be calibrated, limiting the maximum range of maximum acceleration, this article takes the most common value of $3.5 ; \beta_{b}$ is the parameter to be calibrated, which represents the sensitivity of the maximum acceleration followed by the speed change of the vehicle. This article takes the most common value of $0.0875 ; v_{n}$ indicates the instantaneous speed of the rear vehicle.

\subsubsection{Crash Danger Area and Brake Collision Avoidance Area}

When the follow-up distance between the following vehicle and the front vehicle is less than the braking distance, the car enters the crash danger area. When the distance between the rear vehicle and the front vehicle is less than the safe braking distance calculated based on the current speed difference, that is, when entering the brake collision avoidance area, the rear vehicle driver takes the emergency brake and decelerates with $b_{n}$.

$$
\begin{gathered}
b_{n}=\frac{1}{2} \cdot \frac{(\Delta v)^{2}}{A B X-\left(\Delta v-L C_{n-1}\right)}+b_{n-1}+b_{\min } \cdot \frac{A B X-\left(\Delta v-L C_{n-1}\right)}{B X} \\
b_{\min }=\psi_{b}+\sigma_{b} \cdot v_{n}
\end{gathered}
$$

Where, $L C_{n-1}$ indicates the length of the front car; $\psi_{b}$ is the parameter to be calibrated, which limits the upper limit of maximum acceleration during deceleration. This paper takes the most common value of $-20 ; \sigma_{b}$ is the parameter to be calibrated, which represents the sensitivity of the maximum acceleration followed by the speed change of the vehicle during deceleration. This paper takes the most common value of $0.025 ; v_{n}$ indicates the instantaneous speed of the rear vehicle.

\section{Results and Discussions}

The modern community has various structures, in which the block, the axial layout, centripetal layout and intensive type are normal. The internal roads of communities with different structures have their own characteristics, thus the influences on capacity of surrounding main roads are different. In this paper, we take Shenzhen as an example to simulate and analyze traffic changes before and after the opening of residential area.

\subsection{The Block Layout}

We take the community with block layout in Shenzhen as an example. The block layout refers to the residential area divided by blocks, that is, some residential buildings get together, leaving a corresponding road between each block. The figure 2 below shows the specific structure. 


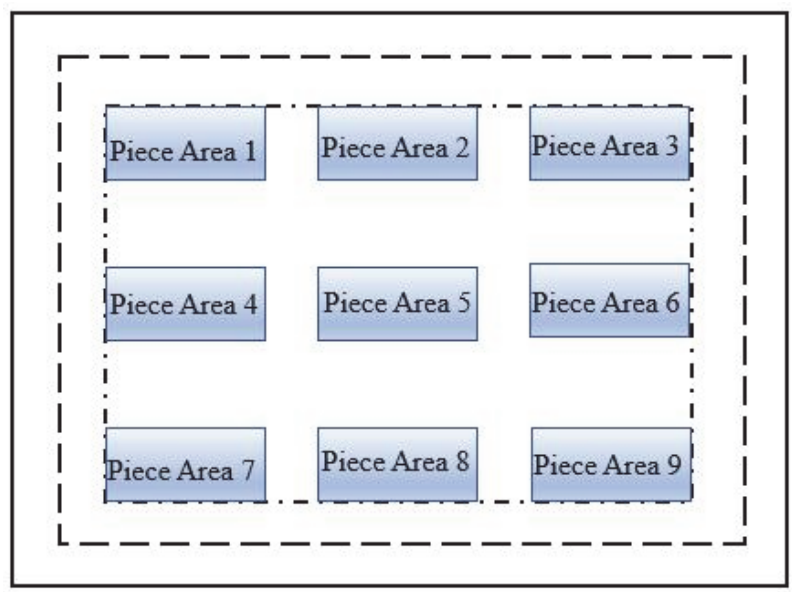

Figure 2. The structure diagram of block-layout communities

According to structure surrounding the community, the roads around the community are set as the most common two-way single lanes, and the roads within the community are set as single lanes as their narrowness. At the same time, we assume the total volume of traffic entering the area was 2.67 units/S; moreover, the traffic flow detectors and average speed detectors are placed on the main roads around the community. The detector takes 20 seconds as the sampling interval, and then according to the calculation formula of the average speed, we can calculate the average speed of the surrounding road vehicles before and after the opening of the community. Similarly, we set traffic light, delay detector and queue length detector at all intersections. And then applying the average delay time model and average queue length model, we can get the mean delay time and queue length of surrounding roads before and after opening the community. Finally, by simulating, all evaluation indicators are got.

After the basic parameters are set, we simulate the vehicle driving rules using the Wiedemann car-following model. The following table 1 shows the simulation results.

Table 1. Comparison of Evaluation Indexes before and after Opening block-layout Residential Area

\begin{tabular}{cccc}
\hline indicators & $\begin{array}{c}\text { Before opening the } \\
\text { community }\end{array}$ & $\begin{array}{c}\text { After opening the } \\
\text { community }\end{array}$ & $\begin{array}{c}\text { The improved } \\
\text { percentage }\end{array}$ \\
\hline Average speed $(\mathrm{km} / \mathrm{h})$ & 30.29 & 31.80 & $4.99 \%$ \\
Average delay time(s) & 17.71 & 8.58 & $51.55 \%$ \\
The queue length(m) & 70.68 & 59.53 & $15.78 \%$ \\
The traffic & 1.52 & 1.59 & $4.61 \%$ \\
flow(unit/s) & & & \\
\hline
\end{tabular}

From the above table 1, we can see that road traffic has been greatly improved after opening the block-type community. More specifically, the average delay time was the most significant improvement (up to 51.55\%), the queue length improvement was large (to $15.78 \%$ ), while the average speed and traffic flow did not increase significantly (both less than $5 \%$ ). 

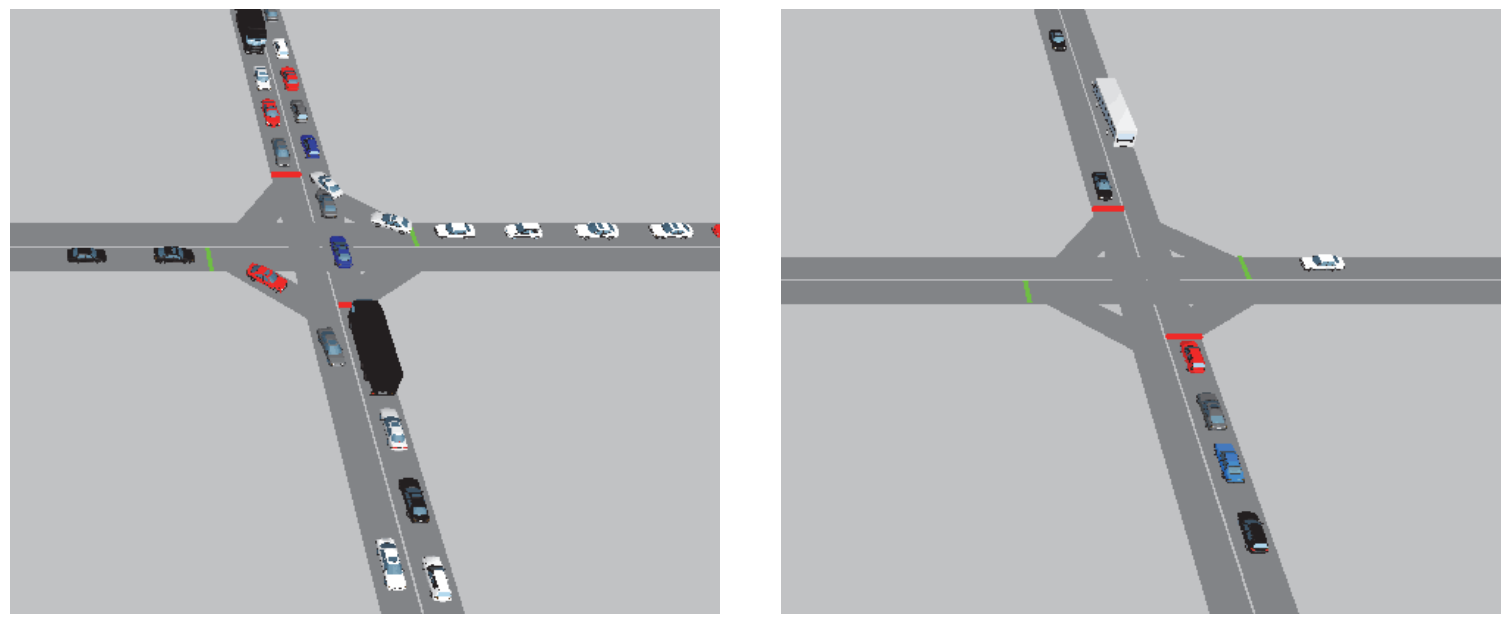

Figure 3. The Observed Images comparison of intersections at closed community (Left) and Open (Right) community

The above figure 3 shows the traffic conditions at the intersection before and after opening the cell, from which we can directly see the improvement of congestion. When opening the community, internal roads are used for traffic, and the vehicle's driving paths are more diversified, which also avoids the congestion at the intersection lights, thereby reducing the average delay time and shortening the queue length.

The average speed and vechicle flow have been improved $4.99 \%$ and $4.61 \%$ respectively, which are not significant. This may be related to the speed at which vehicles will slow down at the intersection. And the number of vehicles on the road and the traffic density are still large after the opening According to the calculation formula of the traffic volume, it can be seen that when the average speed does not increase much, the traffic volume does not substantially improve.

\subsection{The Axial Layout}

In an axis-type layout community, the floor area is arranged along the axis, or symmetrical or balanced, forming a spatial sequence with rhythm. The road distribution diagram is shown in Figure 4 below.

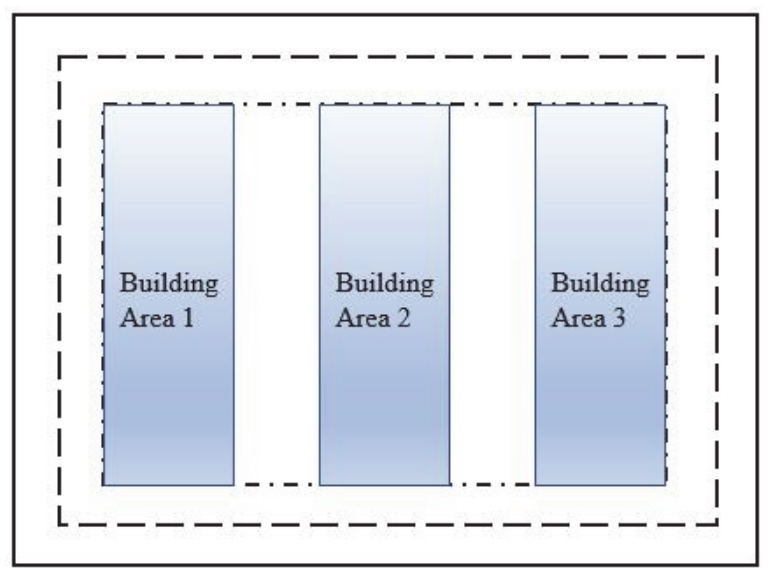

Figure 4. The structure diagram of axis-layout communities

Similarly, according to the structures surrounding the community, we deploy a similar traffic network on the VISSIM software, and the simulation was performed using the vehicle driving rules of the Wiedemann carfollowing model. We can get the average speed, average delay time, the queue length and traffic flow et al. the following table 2 shows the results. 
Table 2. Comparison of Evaluation Indexes before and after Opening axis-layout Residential Area

\begin{tabular}{cccc}
\hline indicators & $\begin{array}{c}\text { Before opening the } \\
\text { community }\end{array}$ & $\begin{array}{c}\text { After opening the } \\
\text { community }\end{array}$ & $\begin{array}{c}\text { The improved } \\
\text { percentage }\end{array}$ \\
\hline Average speed $(\mathrm{km} / \mathrm{h})$ & 30.29 & 31.02 & $2.41 \%$ \\
Average delay time(s) & 17.71 & 10.56 & $40.37 \%$ \\
The queue length(m) & 70.68 & 62.48 & $11.60 \%$ \\
The traffic & 1.52 & 1.56 & $2.63 \%$ \\
flow(unit/s) & & & \\
\hline
\end{tabular}

It can be seen from table 2 that the improvement of surrounding road traffic conditions after the opening of the axis type cell is obvious. The percentage of improvement in the average delay time was the highest (40.37\%), followed by the queue length (reaching $11.60 \%$ ), while the improvement of average speed and traffic flow was not significant (both less than $3 \%$ ).

\subsection{The Centripetal Layout}

The centripetal layout of the residential road structure is that the surrounding road vehicles can enter the inner area of the community in multiple directions, and the end point of the community road converges in an annular area. The schematic diagram is shown in Figure 5.

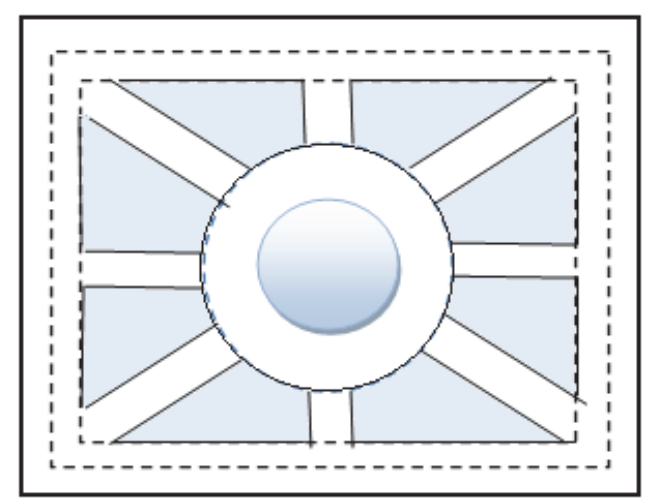

Figure 5. The structure diagram of centripetal layout communities

Similarly, according to the structures surrounding the community, we deploy a similar traffic network on the VISSIM software, and the simulation was performed using the vehicle driving rules of the Wiedemann carfollowing model. We can get the average speed, average delay time, the queue length and traffic flow et al. the following table 3 shows the results.

Table 3. Comparison of Evaluation Indexes before and after Opening centripetal layout Residential Area

\begin{tabular}{cccc}
\hline indicators & $\begin{array}{c}\text { Before opening the } \\
\text { community }\end{array}$ & $\begin{array}{c}\text { After opening the } \\
\text { community }\end{array}$ & $\begin{array}{c}\text { The improved } \\
\text { percentage }\end{array}$ \\
\hline Average speed(km/h) & 30.29 & 32.82 & $8.35 \%$ \\
Average delay time(s) & 17.71 & 8.19 & $53.75 \%$ \\
The queue length(m) & 70.68 & 45.48 & $35.65 \%$ \\
The traffic & 1.52 & 1.76 & $15.79 \%$ \\
flow(unit/s) & & & \\
\hline
\end{tabular}

From the above table 3, we can find that compared with the block layout and axial layout, the opening of centripetal-layout communities has a more significant improvement on the traffic of surrounding roads. The percentage of improvement in the average delay time exceeded half (reaching 53.75\%), while the length of the queue was greatly reduced (improved by $35.65 \%$ ), and the average speed and traffic flow were also improved significantly (both higher than $8 \%$ ). 


\subsection{The Intensive Layout}

The community with intensive layout is characterized by dense facilities, road congestion and inconvenient traffic. There are only a small number of cars in the community barely able to pass through the narrow lanes, and because there are more residents, the traffic speed is slower. The following figure6 shows the specific structure.

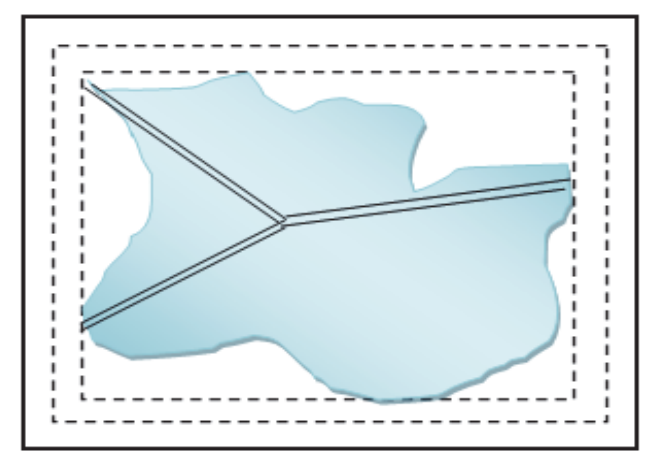

Figure 6. The structure diagram of intensive layout communities

Similarly, according to the structures surrounding the community, we deploy a similar traffic network on the VISSIM software, and the simulation was performed using the vehicle driving rules of the Wiedemann carfollowing model. We can get the average speed, average delay time, the queue length and traffic flow et al. the following table 3 shows the results.

Table 4. Comparison of Evaluation Indexes before and after Opening intensive layout Residential Area

\begin{tabular}{cccc}
\hline indicators & $\begin{array}{c}\text { Before opening the } \\
\text { community }\end{array}$ & $\begin{array}{c}\text { After opening the } \\
\text { community }\end{array}$ & $\begin{array}{c}\text { The improved } \\
\text { percentage }\end{array}$ \\
\hline Average speed(km/h) & 30.29 & 29.42 & $-2.87 \%$ \\
Average delay time(s) & 17.71 & 17.26 & $2.54 \%$ \\
$\begin{array}{c}\text { The queue length(m) } \\
\text { The traffic } \\
\text { flow(unit/s) }\end{array}$ & 70.68 & 68.48 & $3.11 \%$ \\
\hline
\end{tabular}

From the above table4, we can see that opening of intensive community has less impact on the delay time and queue length of vehicles at the intersection, and the improvement percentages are $2.54 \%$ and $3.11 \%$ respectively. However, the average speed and traffic volume have been reduced, and the percentage increases are $-2.87 \%$ and $1.97 \%$ respectively. This means that the opening of intensive communities will lead to a decrease in the average speed and traffic volume of surrounding roads. Through analyzing, we find that this is mainly related to the intensive residential buildings and densely distributed facilities. Even if such roads are opened inside the community, traffic entering outside the community will also block traffic with a high probability, and pedestrians will be distributed along the roads, which will also affect the traffic capacity to a certain extent. In a word, the opening of intensive community will affect the capacity of the main road.

\section{Conclusion}

With the rapid development of urban construction in China, the traffic problem of urban roads has become increasingly severe, and large scale gated residential communities have destroyed urbans' road network structure, which is an important factor causing urban traffic congestion. This article discusses whether opening communities can optimize road network structure, improve road capacity and traffic conditions or not. First of all, we select the four indicators of average speed, vehicle flow, average delay time, queue length to measure traffic capacity. Then we establish the Wiedemann car-following model and use VISSIM software to simulate the traffic capacity of the surrounding roads before and after opening communities. Finally, taking into account the different characteristics of internal roads within communities, we use Shenzhen as an example to study the four types of areas: intensive, 
axis, centripetal, and intensive layout. Through simulation, analysis, and comparison, it is found that in addition to the community structure with the intensive layout, the communities of other structures can significantly improve the traffic conditions of surrounding roads after opening.

We innovatively applied the Wiedemann car-following model to road traffic of different structures. Using VISSIM traffic simulation software, we simulated the traffic conditions of surrounding roads before and after opening communities and verified the feasibility of opening gated communities. This paper discusses four different types of communities, covering most of the types of gated residential communities in China. Not only has certain promotional value, but also can provide a quantitative scientific basis for urban planning departments and traffic management departments on decision-making.

\section{References}

Akgün, A. A., \& Baycan, T. (2012). Gated communities in istanbul: the new walls of the city. Town Planning Review, 83(1). http://doi.org/10.3828/tpr.2012.5

Bao, X. P., \& Zhang, X. M. (2016). The City's Injuries in Closed Residential Areas. Huazhong Architecture, 11, 36-38. http://doi.org/10.3969/j.issn.1003-739X.2016.11.009

Gao, Y. B. (2004). Analysis of computational model for signalized intersection delays. Transportation Science and Technology, 6(6), 49-51. http://doi.org/10.3969/j.issn.1008-5696.2004.06.023

He, L., \& Lan, Y. (2017). The Impact of Closed Residential Traffic on Urban Traffic. Computer System Applications, 26(11), 260-265. http://doi.org/10.15888/j.cnki.csa.006094

Li, J., \& He, L. (2007). The Close and Openness of Residential Areas: Interpretation of the "Central Garden" and "Fenghua Tiancheng" Residential Quarters. New Construction, 1, 93-96. http://doi.org/10.3969/j.issn.10003959.2007.01.021

Li, M., Song, G. H., Yu, L., \& Zhang, Y. H. (2013). Feasibility analysis of road traffic emissions assessment based on vissim. Transportation Systems Engineering and Information, 13(5), 56-63. http://doi.org/10.3969/j.issn.1009-6744.2013.05.009

Li, X. P. (2014). Urban Traffic Congestion Countermeasures - Research on Closed Community Traffic Opening. (Doctoral dissertation, Changsha University of Science and Technology). http://doi.org/10.7666/d.Y2756112

Li, X. Q., Zhu, C. B., Li, D. M., \& Chen, K. (2017). Study on the Impact of Community Opening on the Capacity of Surrounding Roads. Journal of Hebei North University: Natural Science Edition, 33(9). http://doi.org/10.3969/j.issn.1673-1492.2017.09.008

Liu, Lisha, \& Wang, Ya. (2006). Traffic simulation based on vissim. Traffic Science and Technology, 3, 79-81. http://doi.org/10.3963/j.issn.1671-7570.2006.03.028

Qiang, N. Sh. (2016). The Closed Residential Community in the Perspective of Spatial Dialectics and Its Problems. Exploration and Contention, 1(11), 71-75. http://doi.org/10.3969/j.issn.1004-2229.2016.11.017

Tang, K. (2017). Legal path of community roads in the context of "block system"-From the viewpoint of public traffic easements. Journal of Chongqing University of Technology (Social Sciences), 10, 115-125.

Wang, Ch. X., Wu, Q. Sh., Jiao, B. X., \& Zhong, D. Q. (2017). Comprehensive Analysis of Traffic Delays in the Design of Traffic Management and Control Courses. Laboratory Research and Exploration, 36(5), 224-227. http://doi.org/10.3969/j.issn.1006-7167.2017.05.054

Wei. (2016). Open Road to Closed Residential Areas. Urban Architecture, 22, 35-38. http://doi.org/10.3969/j.issn.1673-0232.2016.22.007

Xu, M., \& Yang, Zh. (2010). Super Block + Access Control Community: The Death of Urban Public Space. Journal of Architectural Engineering, 3, 12-15. http://doi.org/10.3969/j.issn.0529-1399.2010.03.003

\section{Copyrights}

Copyright for this article is retained by the author(s), with first publication rights granted to the journal.

This is an open-access article distributed under the terms and conditions of the Creative Commons Attribution license (http://creativecommons.org/licenses/by/4.0/). 